SANTANA, A.F. et al. Eficiência do nitrato de prata a $4 \%$ e da oxitetraciclina spray no controle da ceratoconjuntivite em caprinos e ovinos no semi-árido baiano. PUBVET, Londrina, V. 5, N. 29, Ed. 176, Art. 1189, 2011.

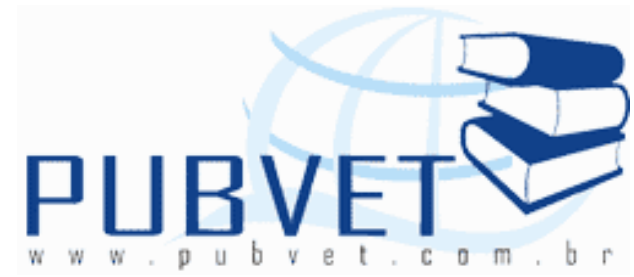

PUBVET, Publicações em Medicina Veterinária e Zootecnia.

\title{
Eficiência do nitrato de prata a $4 \%$ e da oxitetraciclina spray no controle da ceratoconjuntivite em caprinos e ovinos no semi-árido baiano
}

Adelmo Ferreira de Santana ${ }^{1}$, Viviane Rodrigues Magalhães ${ }^{2}$, Catarina Nunes Bittencourt ${ }^{2}$, Isabelle Pryscylla da Silva ${ }^{3}$

${ }^{1}$ Professor do Departamento de Produção Animal da EMEV-UFBA, ${ }^{2}$ Médica Veterinária Especialista em Produção de Pequenos Ruminantes, ${ }^{3}$ Acadêmica de graduação da EMEV-UFBA.

\section{Resumo}

Foi detectado um surto de Ceratoconjuntivite Infecciosa (CCI), no município de Central, Estado da Bahia, em 33 ovinos e 47 caprinos de um rebanho de 132 animais incluindo ovinos e caprinos. Os animais foram examinados clinicamente antes de serem medicados e o grau de inflamação de cada bulbo ocular foi classificado conforme os escores, sendo assim separados de acordo com grau de inflamação e divididos em dois grupos: o grupo I, foi tratado com nitrato de prata $\left(\mathrm{AgNO}_{3}\right)$ a $4 \%$ sendo administrado uma gota no olho afetado; e o grupo II foi tratado com oxitetraciclina* spray por três dias em ambos tratamentos. O nitrato de prata a $4 \%$ e o cloridrato de oxitetraciclina mostraram-se eficazes no tratamento de ceratoconjuntivite infecciosa dos ovinos e caprinos.

Palavras-chave: Ceratoconjutivite, nitrato de prata, oxitetraciclina, caprinos, ovinos 
SANTANA, A.F. et al. Eficiência do nitrato de prata a $4 \%$ e da oxitetraciclina spray no controle da ceratoconjuntivite em caprinos e ovinos no semi-árido baiano. PUBVET, Londrina, V. 5, N. 29, Ed. 176, Art. 1189, 2011.

\title{
Efficiency of silver nitrate to $4 \%$ and in control of spray oxytetracycline keratoconjunctivitis in goats and sheep in semi-arid of Bahia
}

\begin{abstract}
Detected an outbreak of infectious keratoconjunctivitis (ICC) in the municipality of Central, State of Bahia, 33 sheep and 47 goats from a herd of 132 animals including sheep and goats. The animals were examined clinically before being treated and the degree of inflammation of each eyeball was ranked as the scores, thus separated according to degree of inflammation and divided into two groups: Group I was treated with silver nitrate (AgNO3 ) 4\%, being administered a drop in the affected eye, and group II was treated with oxytetracycline spray $*$ for three days in both treatments. The silver nitrate and $4 \%$ oxytetracycline hydrochloride were effective in the treatment of keratoconjunctivitis in sheep and goats.
\end{abstract}

Keywords: keratoconjunctivitis, silvernitrate, oxytetracycline, goats, sheep.

\section{INTRODUÇÃO}

Nos últimos anos o semi-árido nordestino tem se destacado na exploração de caprinos e ovinos, devido as suas características de grande adaptação às condições adversas em função dos seus hábitos alimentares. Porém, desenvolvimento da caprinovinocultura na região Nordeste do Brasil é severamente afetado por inúmeros fatores, entre eles a alta incidência de doenças principalmente nos sistemas de produção intensivo, devido ao maior contato entre os animais.

A Ceratoconjuntivite infecciosa ovina é uma doença contagiosa, também conhecida por "olho rosado", caracterizada por reação inflamatória aguda da conjuntiva, hiperemia da esclera e pannus, além de aumento de lacrimejamento, blefaroespasmo, fotofobia, descargas oculares purulentas, 
SANTANA, A.F. et al. Eficiência do nitrato de prata a $4 \%$ e da oxitetraciclina spray no controle da ceratoconjuntivite em caprinos e ovinos no semi-árido baiano. PUBVET, Londrina, V. 5, N. 29, Ed. 176, Art. 1189, 2011.

opacidade e ulceração corneana, em casos avançados.. A gravidade varia de um indivíduo para outro, podendo inclusive afetar ambos os olhos, quando acometidos, apresentarem quadros diversos (EGWU et al., 1989; GREIG, 1989; HOSIE e GREIG, 1995). A variação dos sinais clínicos na expressão da doença torna difícil avaliar a extensão das perdas econômicas. Além disso, a ceratoconjuntivite infecciosa é uma doença cosmopolita, sazonal que acomete bovinos, caprinos e ovinos, sem distinção de raça, idade e sexo, embora os animais mais jovens e mais velhos sejam mais susceptíveis. Os animais acometidos desenvolvem imunidade natural que vai diminuindo a partir de dois anos quando podem se infectar novamente (CHAVES et al., 2008).

A doença é mais freqüente na época chuvosa ou quando há o aumento da população de moscas. A transmissão pode ocorrer por contato direto entre os animais doentes e sadios, por moscas ou outros insetos, fômites e pelas mãos dos tratadores. Fatores predisponentes como poeira, gravetos, forragem seca, vento, luz ultravioleta podem lesar superficialmente os olhos dos animais e predispor para o início do processo infeccioso (CHAVES et al., 2008).

As perdas econômicas resultantes da doença são, essencialmente, com os custos medicação, tempo e manejo requerido para o tratamento e perda de peso, além do aumento de toxemia da prenhez e pisoteio das crias por ovelhas cegas (GELLAT, 2003).

No Brasil, existem poucos estudos a respeito de tratamentos alternativos de surtos de ceratoconjuntivite em caprinos e ovinos. Este trabalho tem como objetivo descrever o efeito da solução de nitrato de prata a $4 \%$ e da Oxitetraciclina spray, no tratamento da ceratoconjuntivite em caprinos e ovinos no semi-árido baiano. 
SANTANA, A.F. et al. Eficiência do nitrato de prata a $4 \%$ e da oxitetraciclina spray no controle da ceratoconjuntivite em caprinos e ovinos no semi-árido baiano. PUBVET, Londrina, V. 5, N. 29, Ed. 176, Art. 1189, 2011.

\section{REVISÃO DE LITERATURA}

É caracterizada por uma conjuntivite severa e uma ceratite que podem causar a cegueira temporária e, nos animais mais gravemente acometidos, levar à diminuição da ingestão de alimento, que, nos machos, provoca emagrecimento, e, nas fêmeas, toxemia da prenhez (BRUÉRE, 1993), pois o feto em desenvolvimento requer um consumo energético que a utilização de precursores glicogênicos das reservas maternas não consegue compensar, havendo, portanto, uma grande utilização das reservas de gordura do animal (CAPLE, 1993), traduzindo-se em perdas econômicas significativas para o proprietário.

Outros sinais clínicos, que não os oftálmicos, são descritos em animais acometidos por Chlamydia sp. Incluem-se a poliartrite, principalmente quando cordeiros são afetados (MOORE, 1966) e naqueles com Mycoplasma sp, nos quais a pneumonia pode ser um achado freqüente (MOORE, 1993).

Afetando a conjuntiva e a córnea e às vezes o globo ocular em seu todo,a ocorrência desta patologia esta ligada a outros fatores como cor de pele e pelo em torno dos olhos e, distintos graus de pigmentação das constituintes dos mesmos, características estas de certas raças ovinas e caprinas. Desta forma, tem-se observado, no Nordeste, mais comumente nos ovinos deslanados da raça Santa Inês pelagem branca (cerca de 5 a $10 \%$ do rebanho) e nos caprinos da raça Marota (até $10 \%$ ). Este percentual foi o mesmo encontrado por Baas (1977).

O protocolo de tratamento para a ceratoconjuntivite infecciosa envolve o isolamento em ambiente de pouca luz, repouso e alimentação adequada. O uso de antitérmicos, antissépticos para limpeza ocular, antibióticos locais em forma de colírios ou pomadas, injeções subconjuntivais ou parenterais, midriáticos, substâncias anticolagenolíticas são úteis como tratamento medicamentoso. Vacinas comerciais ou autógenas podem ser utilizadas. Deve ser intensificado 
SANTANA, A.F. et al. Eficiência do nitrato de prata a $4 \%$ e da oxitetraciclina spray no controle da ceratoconjuntivite em caprinos e ovinos no semi-árido baiano. PUBVET, Londrina, V. 5, N. 29, Ed. 176, Art. 1189, 2011.

o combate aos vetores. Recobrimentos conjuntivais sobre as úlceras de córneas, ceratorrafias e enucleações são práticas cirúrgicas que podem ser necessárias, de acordo com a gravidade das lesões (CHAVES, 2004, 1998).

Pela variedade de microrganismos incriminados como desencadeadores da ceratoconjuntivite infecciosa ovina, diversos tratamentos têm sido prescritos, tanto tópicos como sistêmicos. Tanto a oxitetraciclina na apresentação oftálmica como também a neomicina, a polimixina B e os corticóide (MOORE, 1966) tem sido utilizados com sucesso. A oxitetraciclina e a tilosina têm sido indicadas para o tratamento sistêmico (MOORE, 1966 e 1993; RADOSTISTS, 1994). Cefalosporinas foram utilizadas com bons resultados no tratamento da ceratoconjuntivite causada por Brucella ovis em caprinos. A sensibilidade "in vitro" da Brucella ovis à penicilina, à ampicilina, à eritromicina, à oxitetraciclina e à enrofloxacina foi demonstrada por Wang (1996).

\section{MATERIAIS E MÉTODOS}

Foram utilizados 33 ovinos e 47 caprinos de um rebanho com 132 animais do município de Central (BA) localizado na microrregião de Irecê- Bahia. Os animais eram mantidos em sistema extensivo em pastagem nativa e com água e sal mineral à vontade.

Após o aparecimento do surto, os animais foram separados de acordo com o grau de inflamação e divididos em dois grupos, o grupo I foi tratado com nitrato de prata (AgNO3) a $4 \%$ sendo administrado 1 gota no olho afetado, sendo que o grupo II recebeu tratamento com oxitetraciclina spray (TerraCortril $\circledast$ Phizer $\circledast)^{*}$.

Foram tratados 80 animais com apresentação clínica de Ceratoconjuntivite infecciosa em graus distintos de inflamação, comparando-se os tratamentos com oxitetraciclina spray (Terra-cortril@) e Nitrato de prata a $4 \%$. 
SANTANA, A.F. et al. Eficiência do nitrato de prata a $4 \%$ e da oxitetraciclina spray no controle da ceratoconjuntivite em caprinos e ovinos no semi-árido baiano. PUBVET, Londrina, V. 5, N. 29, Ed. 176, Art. 1189, 2011.

O grau de inflamação do globo ocular foi dividido em: grau 1 (lacrimejamento e ligeira hiperemia no globo ocular); grau 2 (lacrimejamento e hiperemia acentuada); grau 3 (lacrimejamento e hiperemia acentuada com formação de película esbranquiçada); e grau 4 (lacrimejamento, hiperemia acentuada e perda da visão).

\section{RESULTADOS E DISCUSSÃO}

Em trabalho realizado de Rezende Et al. (2009) no estado de Goiás em 77 ruminantes, dos municípios de Morrinhos e Jataí e florfenicol (Nuflor ${ }^{\circledR}$, Intervet Schering-Plough) em cada olho afetado, em dose única, via intrapalpebral. A escolha da via intrapalpebral para a administração do florfenicol mostrou-se eficiente, já que houve a rápida recuperação da totalidade dos animais tratados e sem haver danos a nenhuma estrutura dos olhos.

Segundo MOORE (1996), o tratamento sistêmico confere maior facilidade de administração dos fármacos que quando em aplicações tópicas com pomadas e sprays; o seu melhor efeito é dado à melhor difusão do fármaco por todos os tecidos do animal, permitindo metabólitos ativos por maior tempo.

Comparando-se o tratamento proposto aos protocolos convencionais, com pomadas ou sprays oftálmicos, há vantagens práticas e na velocidade de cura. Quando comparado ao uso de fármacos via sistêmica, a aplicação intrapalpebral de medicamentos para tratamento da Ceratoconjuntivite infecciosa apresenta-se economicamente bastante atrativa.

O conjunto de medidas higiênicas, terapêuticas e profiláticas - como o isolamento dos animais doentes, controle de moscas, o uso da solução de $\mathrm{NaCl}$ $0,9 \%$ para a lavagem dos olhos, o cloridrato de tetraciclina em forma de pomada oftálmica e a aplicação de vacina comercial contra a ceratoconjuntivite 
SANTANA, A.F. et al. Eficiência do nitrato de prata a $4 \%$ e da oxitetraciclina spray no controle da ceratoconjuntivite em caprinos e ovinos no semi-árido baiano. PUBVET, Londrina, V. 5, N. 29, Ed. 176, Art. 1189, 2011.

infeciosa são medidas adotadas para os ovinos com ceratoconjutivite infecciosa, fazendo parte do protocolo de tratamento preconizado por CHAVES \& ACIPRESTE (1998), CHAVES \& ACIPRESTE (2004) e CHAVES (2004).

As tabelas 1 e 2 demonstram a utilização de 3 dias para os tratamentos com o nitrato de prata a $4 \%$ e com oxitetraciclina spray (Terra-Cortril $®$ Phizer $®$ ), levando em consideração os quatro graus de inflamação.

No trabalho realizado, os animais que obtiveram melhora clínica apenas no primeiro dia de tratamento não foram repetidas as aplicações dos medicamentos no segundo e no terceiro dia, e os animais que obtiveram melhora clínica no segundo dia de tratamento não foram repetidas as medicações no terceiro dia. Os animais restantes foram necessários três dias de tratamento em virtude da persistência de alterações na córnea, opacidade intensa, ulceração e formação de neovasos.

Tabela 1: Relação entre o número de tratamentos utilizados em ovinos com diferentes graus de ceratoconjuntivite tratados com solução de nitrato de prata a $4 \%$.

\begin{tabular}{c|c|c|c|c}
\hline Grau de Inflamação & \multicolumn{3}{|c|}{ Número de tratamentos } & Total \\
\hline & $\mathbf{1}$ & $\mathbf{2}$ & $\mathbf{3}$ & \\
\hline $\mathbf{1}$ & $\mathbf{9 ( 5 6 , 2 5 \% )}$ & $\mathbf{7}(43,75 \%)$ & - & $\mathbf{1 6}(100 \%)$ \\
\hline $\mathbf{2}$ & $\mathbf{3 ( 3 7 , 5 \% )}$ & $\mathbf{5 ( 6 2 , 5 \% )}$ & - & $\mathbf{8}(100 \%)$ \\
\hline $\mathbf{3}$ & $\mathbf{2 ( 3 3 , 3 3 \% )}$ & $\mathbf{1}(16,66 \%)$ & $\mathbf{3}(50 \%)$ & $\mathbf{6}(100 \%)$ \\
\hline $\mathbf{4}$ & - & $\mathbf{1}(33,33 \%)$ & $\mathbf{2 ( 6 6 , 6 6 \% )}$ & $\mathbf{3}(100 \%)$ \\
\hline Total & $\mathbf{1 4}(42,42 \%)$ & $\mathbf{1 4}(42,42 \%)$ & $\mathbf{5 ( 1 5 , 1 5 \% )}$ & $\mathbf{3 3 ( 1 0 0 \% )}$ \\
\hline
\end{tabular}


SANTANA, A.F. et al. Eficiência do nitrato de prata a $4 \%$ e da oxitetraciclina spray no controle da ceratoconjuntivite em caprinos e ovinos no semi-árido baiano. PUBVET, Londrina, V. 5, N. 29, Ed. 176, Art. 1189, 2011.

Tabela 2: Relação entre o número de tratamentos utilizados para a cura dos animais com diferentes graus de conjuntivite tratados com Oxitetraciclina spray (Terra-cortrilß).

\begin{tabular}{c|c|c|c|c}
\hline $\begin{array}{c}\text { Grau de } \\
\text { Inflamação }\end{array}$ & \multicolumn{3}{|c|}{ Número de tratamentos } & Total \\
\hline & $\mathbf{1}$ & $\mathbf{2}$ & $\mathbf{3}$ & \\
\hline $\mathbf{1}$ & $\mathbf{1 2}(57,14 \%)$ & $\mathbf{9 ( 4 2 , 8 5 \% )}$ & - & $\mathbf{2 1}(100 \%)$ \\
\hline $\mathbf{2}$ & $\mathbf{6 ( 6 0 \% )}$ & $\mathbf{4}(40 \%)$ & - & $\mathbf{1 0}(100 \%)$ \\
\hline $\mathbf{3}$ & $\mathbf{3 ( 2 5 \% )}$ & $\mathbf{5 ( 4 1 , 6 6 \% )}$ & $\mathbf{4 ( 3 3 , 3 3 \% )}$ & $\mathbf{1 2}(100 \%)$ \\
\hline $\mathbf{4}$ & - & $\mathbf{3}(75 \%)$ & $\mathbf{1}(25 \%)$ & $\mathbf{4}(100 \%)$ \\
\hline Total & $\mathbf{2 1}(44,69 \%)$ & $\mathbf{2 1}(44,69 \%)$ & $\mathbf{5 ( 1 0 , 6 2 \% )}$ & $\mathbf{4 7 ( 1 0 0 \% )}$ \\
\hline
\end{tabular}

No tratamento com o Nitrato de Prata a $4 \%$ foi possível observar que $42,42 \%$ dos bulbos tratados apresentaram resolução clínica no primeiro dia, $42,42 \%$ no segundo dia e $15,15 \%$ no terceiro dia. Os bulbos dos olhos tratados com o Terra-cotril $\circledast$, observou-se que $44,69 \%$ dos apresentaram resolução clínica no primeiro dia, $44,69 \%$ no segundo dia e $10,63 \%$ no terceiro dia.

Conclui-se que o Nitrato de Prata a $4 \%$ e o Terra-cotril $®$ mostrou-se eficaz no tratamento de ceratoconjuntivite infecciosa dos ovinos e caprinos, apresentando uma boa resolução clínica, diminuindo a incidência de reincidivas e facilitando o manejo na propriedade.

O tratamento dos casos clínicos deve começar imediatamente após ter sido diagnosticada a doença como forma de impedir que sua evolução leve a lesões irreparáveis da córnea. Vários antibióticos podem ser usados, devendo sempre estar atento para caso de resistência. A prevenção e controle desta doença têm sido realizados através de práticas de manejo quando se quer diminuir o máximo possível a ação dos vetores (mosca) e quantidade de poeira. É 
SANTANA, A.F. et al. Eficiência do nitrato de prata a $4 \%$ e da oxitetraciclina spray no controle da ceratoconjuntivite em caprinos e ovinos no semi-árido baiano. PUBVET, Londrina, V. 5, N. 29, Ed. 176, Art. 1189, 2011.

encontrada no mercado vacina que, se usada corretamente, pode conferir alguma imunidade aos animais.

A redução dos casos clínicos de CCI em um rebanho está associada à medidas preventivas fundamentadas em práticas que visem controlar os elementos que podem funcionar como fômites ou agentes disseminadores e/ou estressantes, e envolvem a limpeza diária das instalações (por varredura e/ou raspagem); desinfecção completa das mesmas a cada 7 dias após limpeza prévia (preferencialmente com lança-chamas ou "vassoura-de-fogo"); isolamento dos animais apresentando quadro clínico; controle da população de moscas e insetos (principalmente, por meio de limpeza e manejo adequado dos dejetos animais); redução da exposição a irritantes mecânicos (poeira); manejo do pastejo para manter o relvado em alturas mais adequadas; manejo do rebanho baseado nos princípios de bem-estar e a quarentena de animais récemadquiridos por no mínimo 15 dias.

\section{REFERÊNCIAS BIBLIOGRÁFICAS}

BRUĖRE, A.N., WEST, D.M. Eye diseases in: The sheep: health, disease and production. Fundation for continuing education of the New Zealand Veterinary Association, Palmerston North, 1993, p. 240-43.

CAPLE, I.W.; McLEAH, J.G. Pregancy toxemia In: Current veterinary therapy. Food animal practice, 3ed., Philadelphia, W .B. Saunders, 1993, p.312-14.

CHAVES, N. S. T.; ACIPRESTE, C. S..Ceratoconjuntivite infecciosa bovina. Ano III, n. 1, p. 1-4, janeiro, 1998. [informativo]

CHAVES, N. S. T.; ACIPRESTE, C. S. Peste do olho. Cultivar bovinos, Londrina, p. 30-31, jun. 2004.

CHAVES, N. S. T., LIMA, A. M. V. AMARAL, A.V.C. Surto de ceratoconjuntivite infecciosa em ovinos causada por Moraxella spp. no Estado de Goiás, Brasil. Ciência Animal Brasileira , v. 9, n. 1, p. 256-261, jan./mar. 2008.

CHAVES, N. S. T. Olho rosado. In: TAVARES, E. Sanidade. DBO, São Paulo, p. 132-133, out. 2004. [entrevista].

DaMASSA, A. J.; WAKENELL, P. S.; BROOKS, D. L .. Mycoplasmas of goats and sheep. Journal of Veterinary Diagnostic Investigation, v. 4, p. 101-103, 1992.

EGWU, G. O; FAULL , W. B.; BRADBURY, J. M.; CLARKSON, M. J. Ovine infectious keratoconjunctivitis: a microbiological study of clinically unaffected and affected sheep's eyes 
with special reference to Mycoplasma conjunctivae. Veterinary Record, v. 125, p.253-256, 1989.

GELLAT, K. N. Manual de oftalmologia veterinária. São Paulo: Manole, 2003. 594 p.

GREIG, A. Ovine keratoconjunctivitis. An update. In Practice, London, v. 11, p. 110 - 113, 1989.

HOSIE, B. D. \& GREIG, A. Role of oxytetracicline dihidrate in the treatment of Mycoplasmaassociated ovine keratconjunctivitis in lambs. British Veterinary Journal, v.151, p.83$88,1995$.

JONES, G. E.; FOGGIE, A.; SUTHERLAND, A.; HARKER, D. Mycoplasma and ovine keratoconjunctivitis. Veterinary Record, v. 99, p.137- 141, 1976.

MAYER, D.; DEGIORGIS, M. P.; MEIER, W.; NICOLET, J. GIACOMETTI, M. Lesions associated with infectious keratoconjunctivitis in Alpine ibex. Journal Wildlife Diseases. V. 33, p.413419, 1997.

MOORE, C.P. Chlamydial keratoconjunctivitis in sheep. In: SMITH B.P. Large animal internal medicine, 2ed., Saint Louis, Mosby, 1996, p. 1362.

MOORE, C.P., W ALLACE, L.M. Selected eye disease of sheep and goats In: HOWARD, J.L. Current veterinary therapy 3 - food animal practice. Philadelphia: W .B. Saunders, 1993, p. $839-42$.

SAMUELSON, D. A. Ophthalmic anatomy. In: GELLAT, K. N. Veterinary ophthalmology. 3a ed. Philadelphia: Lippincott Williams e Wilkins, 1999, p. $31-150$.

SLATTER, D. Fundamentals of veterinary ophthalmology. 3a ed. Philadelphia: W. B. Saunders, 2001, 640 p.

STADES, F. C.; BOEVÉ, M. H.; NEUMANN, W. et al. Fundamentos de oftalmologia veterinária. São Paulo: Manole, 1999. 204 p.

RADOSTITS, O.M., BLOOD, D.C., GAY, C.C. Diseases caused by rickettsia In: Veterinary Medicine, 8 ed., Londres, Baillière Tindal, 1994, p. 1144-46.

REZENDE, M.L.G.; FIORANTI, M. C. S.; LIMA, F. G.; COSTA, G. L.; HIDASI, H. W.; VIANA, R. B. Uso intrapalpebral de florfenicol no tratamento da ceratoconjuntivite infecciosa bovina, ciência animal brasileira - Suplemento 1, 2009 - Anais do VIII Congresso Brasileiro de Buiatria. Disponível em: <http://www.revistas.ufg.br/index.php/vet/article/view/7705/5466> Acessado em: 25 de outubro de 2010.

W A N G, X.J., ZHANG, J.L., SAN, Q.F., ZHANG, M.L., Diagnosis and treatment of Branhamella ovis infection. Chinese Journal Veterinary Medicine, v. 22, n. 3, p. 27, 1996. 